\title{
The FOXK1-CCDC43 Axis Promotes the Invasion and Metastasis of Colorectal Cancer Cells
}

\author{
Jing Wang ${ }^{a}$ Guangnan Liu ${ }^{a}$ Mengwei Liu ${ }^{a}$ Li Xiang $^{b}$ Yizhi Xiao ${ }^{a}$

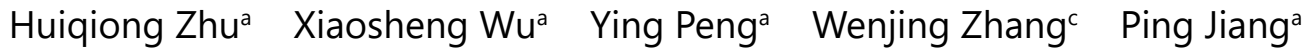 \\ Aimin Lia Qingzhen Nan ${ }^{\mathrm{a}}$ Ye Chen ${ }^{\mathrm{a}}$ Chudi Chen ${ }^{\mathrm{a}}$ Tianming Cheng ${ }^{\mathrm{a}}$ Side Liu ${ }^{\mathrm{a}, \mathrm{b}}$ \\ Jide Wang ${ }^{a, b}$ \\ aGuangdong Provincial Key Laboratory of Gastroenterology, Department of Gastroenterology, Nanfang \\ Hospital, Southern Medical University, Guangzhou, 'DDepartment of Gastroenterology, Longgang \\ District People's Hospital, Shenzhen, 'Department of Medical Oncology, the First people's Hospital of \\ Yunnan Province, Kunming University of Science and Technology, Kunming, China
}

\section{Key Words}

CCDC43 - FOXK1 - Colorectal cancer - Metastasis - Epithelial-mesenchymal transition • Invasion

\begin{abstract}
Background/Aims: The CCDC43 gene is conserved in human, rhesus monkey, mouse and zebrafish. Bioinformatics studies have demonstrated the abnormal expression of CCDC43 gene in colorectal cancer (CRC). However, the role and molecular mechanism of CCDC43 in CRC remain unknown. Methods: The functional role of CCDC43 and FOXK1 in epithelialmesenchymal transition (EMT) was determined using immunohistochemistry, flow cytometry, western blot, EdU incorporation, luciferase, chromatin Immunoprecipitation (ChIP) and cell invasion assays. Results: The CCDC43 gene was overexpressed in human CRC. High expression of CCDC43 protein was associated with tumor progression and poor prognosis in patients with CRC. Moreover, the induction of EMT by CCDC43 occurred through TGF- $\beta$ signaling. Furthermore, a positive correlation between the expression patterns of CCDC43 and FOXK1 was observed in CRC cells. Promoter assays demonstrated that FOXK1 directly bound and activated the human CCDC43 gene promoter. In addition, CCDC43 was necessary for FOXK1- mediated EMT and metastasis in vitro and vivo. Taken together, this work identified that CCDC43 promoted EMT and was a direct transcriptional target of FOXK1 in CRC cells. Conclusion: FOXK1-CCDC43 axis might be helpful to develop the drugs for the treatment of CRC.
\end{abstract}




\section{Cellular Physiology Cell Physiol Biochem 2018;51:2547-2563 and Biochemistry Published \begin{tabular}{l|l} 
DOI: 10.1159/000495924 & $\begin{array}{l}\text { ( } 2018 \text { The Author(s). Published by S. Karger AG, Basel } \\
\text { www.karger.com/cpb }\end{array}$
\end{tabular} \\ Wang et al.: FOXK1 Promotes CCDC43-Mediated CRC Progression}

\section{Introduction}

The coiled-coil motif is found in many proteins, such as skeletal and motor proteins and is involved in molecular recognition systems and protein refolding [1-3]. To date, more than 140 genes contain the coiled-coil domain, but their functions are unknown in many cases (http://www.genecards.org/). Previous studies have shown that approximately 40 CCDC genes are associated with diseases, including various metabolic diseases [4], cancers [5-7], mitochondrial disease [8], epigenetic disease [9] and heart disease [10]. The abnormal expression of CCDC containing proteins in nasopharyngeal carcinoma [7], prostate cancer [5], pancreatic cancer [6], gastric cancer [9], breast cancer [11] and colorectal cancer (CRC). [12] is directly linked to the phenotype of tumor cell migration, invasion and metastasis.

CCDC43 is located on chromosome 17q21.31 and encodes a 224-amino-acid protein containing a coiled-coil domain. Chromosome 17 is rich in protein-coding genes, and has the second highest gene density in the genome. The GENT database shows that CCDC43 mRNA is upregulated in cancers of the ovary, lung, head and neck, cervix, breast, adrenal gland, stomach, liver, esophagus, bladder, prostate, vagina, testis and colon compared with the corresponding normal tissues. However, the molecular mechanism by CCDC43 in human tumor has not been investigated.

The human FOXK1 gene encodes predicted proteins that are most homologous to the mouse myocyte nuclear factor MNF/Forkhead box K1 (Foxk1)[13]. Murine FOXK1 (Foxk1/ MNF) exists as two isoforms, MNFa and MNFb (the C-terminally truncated MNFb isoform is produced through alternative splicing) [14]. For human FOXK1, protein feature analysis predicted the existence of a forkhead domain, a FHA domain, and a nuclear localization sequence (NLS) [15]. Previous studies have suggested that knockdown of FOXK1 eliminates cell cycle-dependent oscillations and results in decreased cell proliferation rates and the development of the malignant phenotype in human osteosarcoma U2OS cells [16]. We recently found that FOXK1 was overexpressed in 16 types of solid tumor tissues via tissue multi-array (TMA) [17]. These data support a role for human FOXK1 in regulating the developmental process as well as the potential involvement of FOXK1 in tumorigenesis. FOXK1 is a transcription factor that modulates target gene expression. Yang et al. have showed that MNF/Foxk1, which mediates its DNA binding, recognizes the DNA sequence motif, WRTAAAAYA and regulates the p21, c-myc [16], or cdc2 gene [18]. However, the mechanism by which FOXK1 regulated CCDC43 expression during the progression and prognosis of CRC remain to be defined.

In this study, we showed that CCDC43 expression was elevated in cancer tissues. For the first time, we demonstrated that CCDC43 overexpression promoted proliferation, invasion and EMT in CRC. Moreover, CCDC43 was involved in TGF- $\beta 1$-induced EMT in CRC cells. Furthermore, FOXK1 regulated CCDC43 expression by transcriptional activation in human CRC cells, thereby promoting cell invasion and metastasis in vitro and in vivo. Thus, the tumor progression and metastasis were mediated by a key signaling pathway involving the FOXK1- CCDC43 axis.

\section{Materials and Methods}

Reagents and culture conditions

Recombinant human TGF- $\beta 1$ (240-B) was purchased from R\&D Systems (Minneapolis, MN, USA). Rabbit anti-CCDC43 (N-15), MMP2 (H-76), CDK4 (DCS-35), CDK6 (B10), Cyclin B1 (G11), Cyclin D1 (A10) and E-cadherin (H-108) were purchased from Santa Cruz (Santa Cruz, CA, USA). Rabbit anti-Vimentin (Ag0489) and mouse anti-human $\beta$-actin were purchased from Proteintech (Wuhan, China).

The human colon cancer cell lines LoVo, HT29, SW480, SW1116, DLD1 and SW620 were all maintained in our laboratory as previously described. The cells were cultured in RPMI 1640 (Life Technologies, Inc., Gaithersburg, MD, USA) supplemented with $10 \%$ fetal bovine serum (FBS), $100 \mu \mathrm{g} / \mathrm{mL}$ streptomycin and $100 \mu / \mathrm{mL}$ penicillin in a humidified incubator at $37^{\circ} \mathrm{C}$ with an atmosphere of $5 \% \mathrm{CO}_{2}$. 


\section{Cellular Physiology Cell Physiol Biochem 2018;51:2547-2563

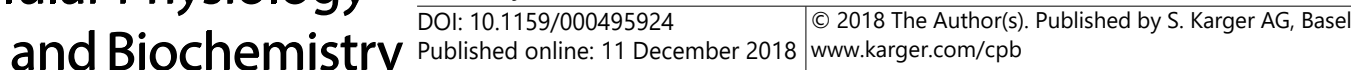

Wang et al.: FOXK1 Promotes CCDC43-Mediated CRC Progression

\section{Tissue multi-array (TMA)}

Tissue microarray (TMA) human CRC tissues and matched non-cancerous colonic tissues were purchased from superchip (Shanghai, China). In the current study, all 82 tissues were adenocarcinomas. The expression of CCDC43 was detected by immunohistochemistry (IHC) in TMA slides described previously. Tumor staging was defined according to the criteria for histological classification proposed by the International Union against Cancer (UICC). Scoring of the tissue slides was performed independently by two investigators; the intensity of the cancer cell staining was scored as 0 (negative), 1 (weak expression, fancy light yellow), 2 (moderate expression, fancy yellow) and 3 (strong expression, fancy intense yellow). An intensity score $\geq 2$ with at least 50\% CCDC43-positive cells was considered high expression (or overexpression), and $<50 \%$ CCDC43-positive cells or an intensity score $<2$ was regarded as low expression. The study was approved by the institutional human ethics committee of the relevant institutions.

\section{Constructs and establishment of stable transfectants}

Normal human complementary DNA (cDNA) corresponding to the full-length CCDC43 was obtained by RT-PCR amplification described previously $[19,20]$. The PCR aliquots were subcloned into the mammalian expression vector pENTER-FLAG (ViGene Biosciences, Rockville, MD, USA).

To establish stable cell lines, cells transfected with empty pENTER vector and pENTER-CCDC43 were passaged at 1:15 (vol/vol) and cultured in RPMI 1640 medium supplemented with puromycin (Sigma, St. Louis, MO, USA) at $2 \mu \mathrm{g} / \mathrm{ml}$ for 4 weeks.

\section{RNA isolation and quantitative real-time RT-PCR}

Cells were harvested, and total RNA was extracted using TRIzol Reagent (Gibco BRL, Gaithersburg, MD, USA). RNA was reverse-transcribed to cDNA using Thermoscript RT system reagent (Gibco BRL) in accordance with the manufacturer's instructions.

Quantitative real-time PCR was performed using an Applied Biosystems Sequence Detection System 7900 (ABI Prism 7900HT, Applied Biosystems Company, USA) with a 10- $\mu$ l mixture composed of Power SYBR GREEN PCR Master Mix (Applied Biosystems, Foster City, CA, USA), $500 \mathrm{nmol}$ of each primer, and $300 \mathrm{ng}$ of cDNA template. The reactions were performed with an initial denaturation at $95^{\circ} \mathrm{C}$ for 5 minutes, followed by 60 cycles of 20 seconds at $94^{\circ} \mathrm{C}, 20$ seconds at $60^{\circ} \mathrm{C}$, and 40 seconds at $72^{\circ} \mathrm{C}$. A final extension at $72^{\circ} \mathrm{C}$ for 5 minutes was included before a temperature ramp from $72^{\circ} \mathrm{C}$ to $95^{\circ} \mathrm{C}$ at $0.1{ }^{\circ} \mathrm{C} / \mathrm{s}$ with continuous fluorescent acquisition. Each cDNA sample was processed in duplicate for each q-RT-PCR assay, and the average relative fold mRNA expression levels were determined using the $2^{-\Delta \Delta C t}$ method with GAPDH as the internal control. The primer sequences used for q-RT-PCR were as follows: Vimentin forward: 5'-GGGACCTCTACGAGGAGGAG-3' and Vimentin reverse: 5'- CGCATTGTCAACATCCTGTC-3' (200 bp); GAPDH forward: 5'- GTCAACGGATTTGGTCGTATT- 3' and GAPDH reverse: CTCCTGGAAGATGGTGATGGG (216 bp).

\section{Gene silencing using siRNA}

The sequences of the CCDC43 siRNA and the Scrambled (Scr) siRNA were as follows (sense strand): 113- CGTTTATGGAGCCTACATC -132, and 5'-CGUACGCGGAAUACUUCGA-3', respectively, as described previously in detail.

\section{EDU cell proliferation assay}

Each group of isolated tumor cells was seeded into 96-well plates in triplicate at a density of $10^{3} /$ well for $48 \mathrm{~h}$ of incubation. The cells were incubated for an additional $2 \mathrm{~h}$ in medium containing $50 \mu \mathrm{M}$ EdU (RiboBio, Guangzhou, China), and were then washed with PBS, fixed and permeabilized with PBS containing $4 \%$ paraformaldehyde and $0.5 \%$ Triton X-100. The cells were incubated with $1 \times$ Apollo reaction cocktail $(100 \mu \mathrm{l} /$ well) for $30 \mathrm{~min}$. The DNA was incubated with Hoechst 33258 stain $(100 \mu \mathrm{l} /$ well) for $30 \mathrm{~min}$ and visualized with an inverted fluorescence microscope (Leica DM5500, Germany). For each EdU experiment, five random fields were imaged at $100 \times$ magnification. Captured images were processed and analyzed with ImageJ software. The number of EdU-positive cells was identified by Hoechst nuclear staining and expressed as a percentage of the total number of cells in each field. 


\section{Cellular Physiology Cell Physiol Biochem 2018;51:2547-2563 and Biochemistry \begin{tabular}{l|l} 
DOI: 10.1159/000495924 & Published 2018 The Author(s). Published by S. Karger AG, Basel \\
www.karger.com/cpb
\end{tabular}}

Wang et al.: FOXK1 Promotes CCDC43-Mediated CRC Progression

\section{Flow cytometry analysis}

For cell cycle analysis, CRC cells were reverse transfected in 6-well dishes as described and incubated for $48 \mathrm{~h}$ under regular growth conditions. Cells were fixed for $90 \mathrm{~min}$ with $70 \% \mathrm{v} / \mathrm{v}$ ) pre-cooled ethanol $\left(-20^{\circ} \mathrm{C}\right)$ and harvested by centrifugation $\left(4^{\circ} \mathrm{C}, 5 \mathrm{~min}, 1,000 \mathrm{~g}\right)$. Staining was performed using a $69 \mu \mathrm{M}$ propidium iodide solution in PBS for $30 \mathrm{~min}$ at $37^{\circ} \mathrm{C}$, including RNaseA $(0.6 \mu \mathrm{g} / \mathrm{ml})$. The cell cycle distribution was calculated from the resultant DNA histogram using FlowJo software.

\section{Western blot analysis and immunofluorescence}

For Western blot analysis, cells were harvested and lysed in lysis buffer. A total of $30 \mu \mathrm{g}$ of protein lysates were separated by SDS-PAGE and transferred onto a PVDF membrane. Primary antibodies were diluted according to the company's recommendation. Protein bands were detected by using ECL Western Blotting Detection Reagent (GE Healthcare). Cells grown on cover glasses were fixed with 4\% paraformaldehyde, and nonspecific binding was blocked by incubation with $1 \%$ bovine serum albumin. The glasses were probed with the primary antibodies followed by TR- or fluorescein isothiocyanate-conjugated secondary antibodies. After mounting, the slips were visualized under an Olympus CKX 41 fluorescence microscope (Olympus Optical Co., Ltd., Tokyo, Japan).

\section{Cell migration and invasion assay}

Invasion and cell migration assays were performed as described [17]. Cells were plated in serumfree medium on Transwell inserts (Corning, NY, USA) coated with $25 \mu \mathrm{g}$ of Matrigel (BD Biosciences) for the invasion assays. After incubation for $48 \mathrm{~h}$ at $37^{\circ} \mathrm{C} / 5 \% \mathrm{CO}_{2}$, the inserts were fixed with $3.7 \%$ paraformaldehyde/PBS and stained with $2 \%$ crystal violet. The number of invading cells was counted in five representative (200x) fields per insert. Cell migration assays were performed. Briefly, cells plated in 6-well plates at $100 \%$ confluence were wounded with a pipette tip at time 0 . The medium was changed to remove cell debris, and the cells were cultured in the presence of $10 \mu \mathrm{g} / \mathrm{ml}$ mitomycin $\mathrm{C}$ to inhibit cell proliferation. Images were captured after $72 \mathrm{~h}$.

\section{Promoter reporter and dual luciferase assays}

The PCR reaction generated an 800-bp product from the CCDC43 proximal promoter (CCDC43p1 and CCDC43p2, the upstream nucleotide adjacent to the transcription start site was defined as 1) containing FOXK1 ( -498 to $-490 \mathrm{bp}$ and -677 to $-669 \mathrm{bp}$ ). The putative CCDC43 promoter region was cloned into the pGL3basic vector and transfected into colorectal cancer cells. The firefly and Renilla luciferase activities were measured using the Dual-Luciferase reporter system (Promega) with a model TD-20/20 Luminometer (EG\&G, Berthold, Australia). The firefly luciferase activity value was normalized to the Renilla activity value. The transcriptional activity at the promoter was presented as the fold induction of relative luciferase units (RLUs) compared with the basic pGL3 vector control. The RLU was the value of the firefly luciferase unit divided by the value of the Renilla luciferase unit. Site-directed mutagenesis of the potential FOXK1 binding site was carried out in the CCDC43p1 plasmid using the ClonExpress II One Step Cloning Kit (Vazyme, Nanjing, China). The primer sequences are listed in Table 1.

\section{Construction and production of recombinant lentivirus}

To further investigate the effects of siRNA-induced downregulation of CCDC43 in FOXK1-overexpressed cells on tumor growth in vivo, an CCDC43-RNAi lentiviral vector (pGCSIL-FOXK1 shRNA) was constructed (Shanghai GeneChem Co, Ltd, Shanghai, China). Double-stranded oligonucleotides encoding human CCDC43-vshRNA were inserted into the short hairpin RNA (shRNA) expression vector pGCSIL (Shanghai GeneChem Co., Ltd.),

and the identities of the clones were verified by sequencing. To establish the FOXK1 overexpressing and CCDC43 knockdown cell lines, lentiviruses expressing FOXK1 Table 1. Primary Primers Used in This Study

\begin{tabular}{lccc}
\hline Experiment & Name & $\begin{array}{c}\text { Position or } \\
\text { orientation }\end{array}$ & Sequence (5'-3') \\
\hline \multirow{2}{*}{ Luciferase construction } & R: & $-6 \sim+12$ & GAAGATCTCCTACCGCAGTGCCTGAC (Bgl II) \\
& L:CCDC43p1-WT & $-507 \sim-484$ & F:5'GGGGTACTTGCCTGTTTATTACTGAGTGC(Kpn I) -3' \\
Site-directed mutagenesis & L:CCDC43p2-WT & $-692 \sim-672$ & F:5'-GGGGTACCCGGCCTGTCTCCTTATCTTT (Kpn I) -3' \\
& L:CCDC43p-MT & $-507 \sim-484$ & F: TTGCCTGTTGAGTGACTGAGTGC \\
& & L: $-577 \sim-557$ & R:GCACTCAGTCACTCAACAGGCAA \\
Chip & Chip 1 & R: $-367 \sim-387$ & GTGGGGTACGCTTCTCAAAG \\
& & CCTCCTGACAGCCTGGAATA \\
& Chip 2 & L:-719 $\sim-699$ & GGGATTACAGGCGTGAGACA \\
& R:-509 $\sim-533$ & TCAAGTTATTTCTTCCACTCATTG \\
\hline
\end{tabular}




\section{Cellular Physiology Cell Physiol Biochem 2018;51:2547-2563

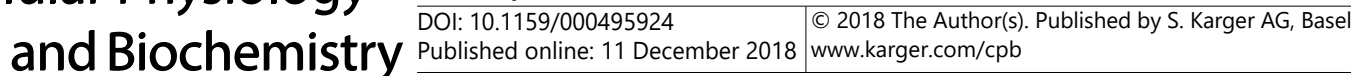 \\ Wang et al.: FOXK1 Promotes CCDC43-Mediated CRC Progression}

cDNA and CCDC43 shRNA or scrambled (Scr) shRNA were co-transfected into the LoVo cell line. All lentiviruses were then mixed with $5 \mu \mathrm{g} / \mathrm{mL}$ polybrene to enhance the transfection efficiency.

\section{In vivo assays for tumor metastasis}

To evaluate the metastatic potential of cancer cells to the liver in vivo, 5X106 LoVo/EGFP-N1, LoVo/ EGFP-FOXK1 scr shRNA and LoVo/EGFP- FOXK1-CCDC43 shRNA cells were injected into the spleens of orthotopic mice ( $n=3$ for each group). One month later, the mice were sacrificed, and the individual organs were removed and assessed using the In vivo F Imaging System (Kodak). The metastatic tissues were analyzed with H\&E, IHC and qRT-PCR.

\section{Statistical analyses}

Statistical analyses were performed using the SPSS statistical software package (standard version 13.0; SPSS, Chicago, IL, USA). Quantitative data obtained from experiments with biological replicates are shown as the means $( \pm$ S.D. or S.E.M). Linear regression and Pearson's correlation analysis were performed to assess the relationship between FOXK1 and CCDC43 in the tissue. Survival analyses were performed using the Kaplan-Meier and log-rank test. Probability values from the two-tailed test $<0.05$ were considered to be significant.

\section{Results}

Colorectal cancer cells expressed higher levels of CCDC43

In the GENT database, CCDC43 is upregulated in cancers of the adrenal gland, bladder, breast, esophagus, head neck, liver, lung, skin, prostate, vagina, uterus, stomach and colon compared with the corresponding normal tissues (Fig. 1). This finding suggests that CCDC43 may be associated with various types of cancer, including colon cancer.

Next, we confirmed the expression of CCDC43 by IHC analysis of the tissue microarray (TMA) containing 82 cases of CRC and adjacent normal tissues. The results showed that CCDC43-positive signals were presented in the cytoplasm and nucleus of cancer cells of $74.4 \%(61 / 82)$ of CRC samples, as exemplified in Fig. 2A. By contrast, normal colon tissues did not express the CCDC43 protein.

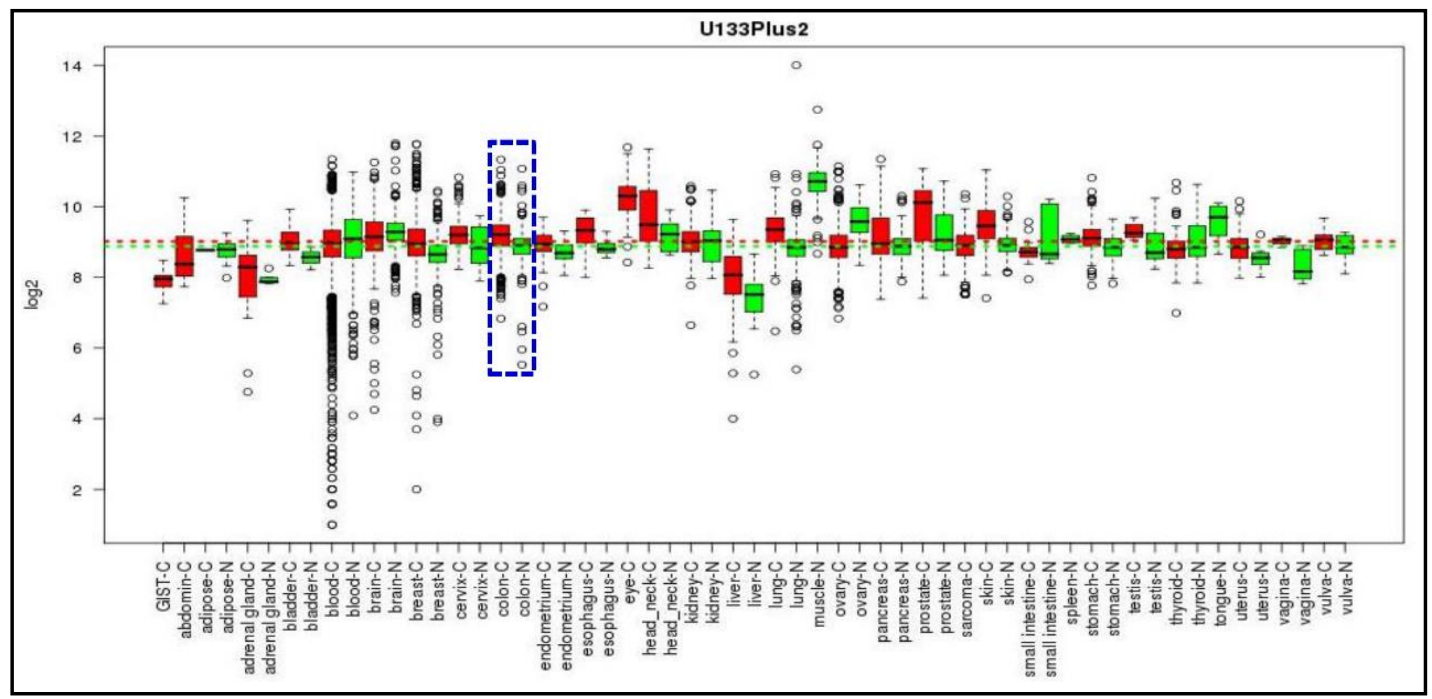

Fig. 1. Expression pattern of CCDC43 mRNA in normal and tumor tissues. CCDC43 mRNA expression in various types of cancer was analyzed in the GENT database. Red boxes represent tumor tissues; green boxes represent normal tissues. Red and green dashed lines represent the average value of all tumor and normal tissues, respectively. The asterisk indicates a significant increase in CCDC43 expression in colon tumors compared with normal tissues. CCDC43 mRNA expression in colon tissue: blue dotted lines. 


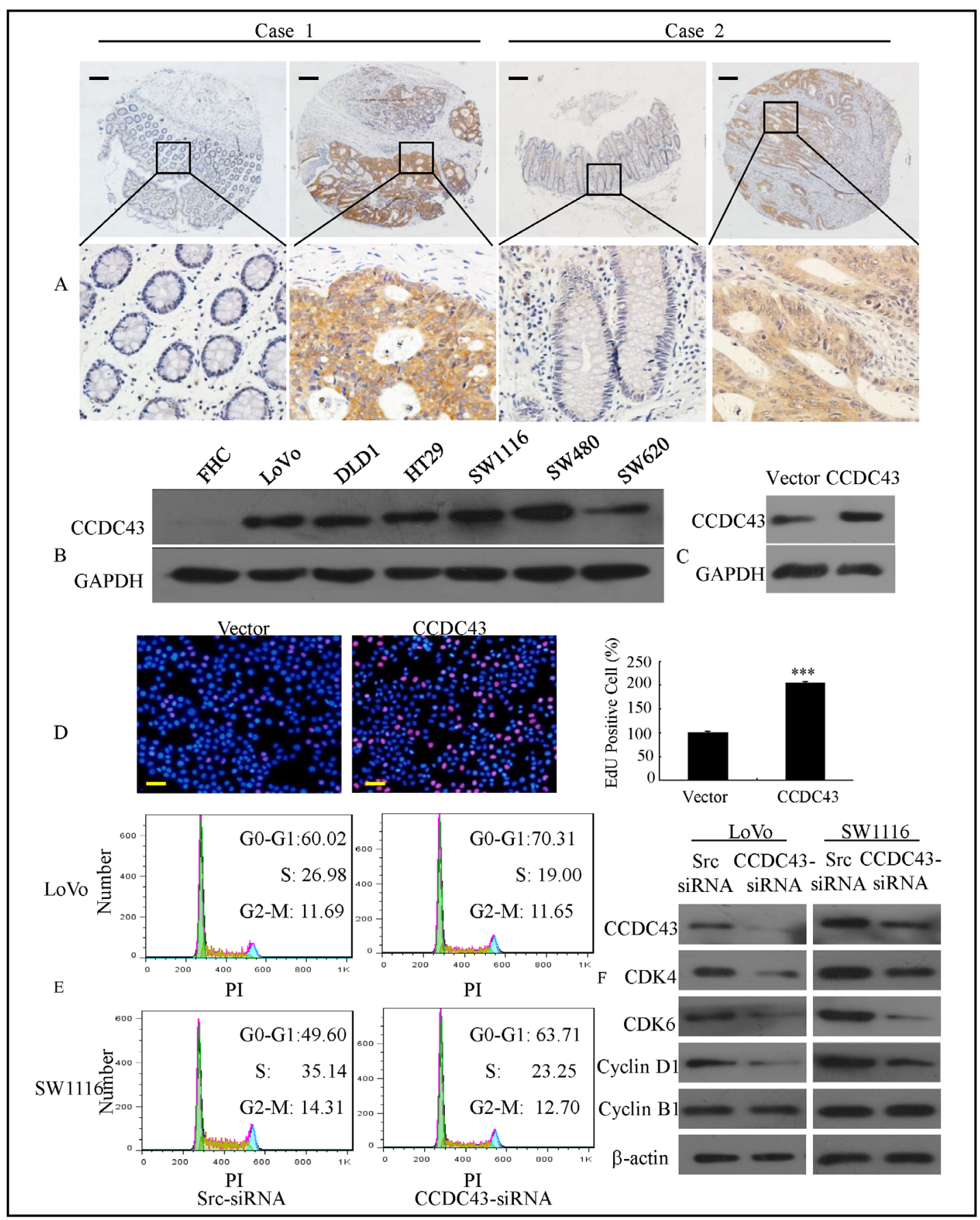

Fig. 2. Over-expression of CCDC43 is associated with cell proliferation in CRC. (A) CCDC43 expression in normal and malignant human colorectal tissues was detected by TMAs. (B) Whole lysates of FHC, LoVo, DLD1, HT29, SW1116, SW480 and SW620 were collected, and CCDC43 was detected by Western blot analysis. GAPDH was used as the internal control (GAPDH: glyceraldehyde-3- phosphate dehydrogenase). (C) CCDC43 pooled stable transfectants and vector control transfectants were detected by Western blot analysis in LoVo cells. (D) DNA synthesis of LoVo cells was measured using an EdU incorporation assay after the indicated transfection at $48 \mathrm{~h} .{ }^{* * *} \mathrm{P}<0.01$, between CCDC43 and vector. (E) The effects of CCDC43-siRNA and scr-siRNA on CCDC43 expression and on the cell cycle distribution of CRC cells were determined by flow cytometry after staining with PI. (F) Whole-cell lysates of parental CRC cells were prepared, and protein expression was detected by Western blotting. The images are representatives of 3 independent experiments with identical results. Scale bars, $50 \mu \mathrm{m}$ in A and $100 \mu \mathrm{m}$ in D.

\section{KARGER}


We then showed increased CCDC43 expression in the following six CRC cell lines: LoVo, DLD1, HT29, SW1116, SW480 and DLD1, compared with the normal colon cell line (FHC) (Fig. 2B).

These findings demonstrated that CCDC43 gene was overexpressed in CRC cells and tissues.

High expression of protein CCDC43 was associated with proliferation in CRC

To assess the effect of ectopic expression of CCDC43 on the malignant behavior of CRC cells in vitro, we established stable transfectants with CCDC43-sense and vector plasmids. We confirmed the CCDC43 expression levels by Western blotting analysis (Fig. 2C). The results showed that overexpression of CCDC43 significantly promoted cell proliferation compared with the control cells (transfected with the vector control; Fig. 2D) by EdU assay.

To determine the mechanism by which CCDC43 downregulation may affect cell growth, cells were infected with CCDC43-siRNA or scrambled-siRNA, and cell cycle analysis was performed. CCDC43 knockdown increased the proportion of cells in the G0/G1 phase and concomitantly decreased the proportion of cells in the $\mathrm{S}$ phase of the cell cycle by flow cytometry (Fig. 2E).

Next, cell cycle-related protein expression was assessed by western blotting. Consistent with the accumulation of cells in G0/G1 phase, Cyclin D1, CDK4 and CDK6 expression levels were significantly decreased in CCDC43-siRNA-treated LoVo and SW1116 cells, whereas Cyclin B1 remained unchanged, compared with the scrambled (Scr)-siRNA-treated cells (Fig. 2F).

Together, these results indicated that CCDC43 silencing in CRC cells was associated with a block in cell cycle progression.

Increased CCDC43 expression correlated with tumor progression and poor prognosis of CRC patients

Elevated cell migration and invasion are associated with an increase in the metastatic potential of cancer cells [21]. This phenomenon may be independent of cell proliferation rates. Therefore, we studied the effect of CCDC43 overexpression on the migration and invasion of CRC cells. The wound healing assay indicated that CCDC43 overexpression led to

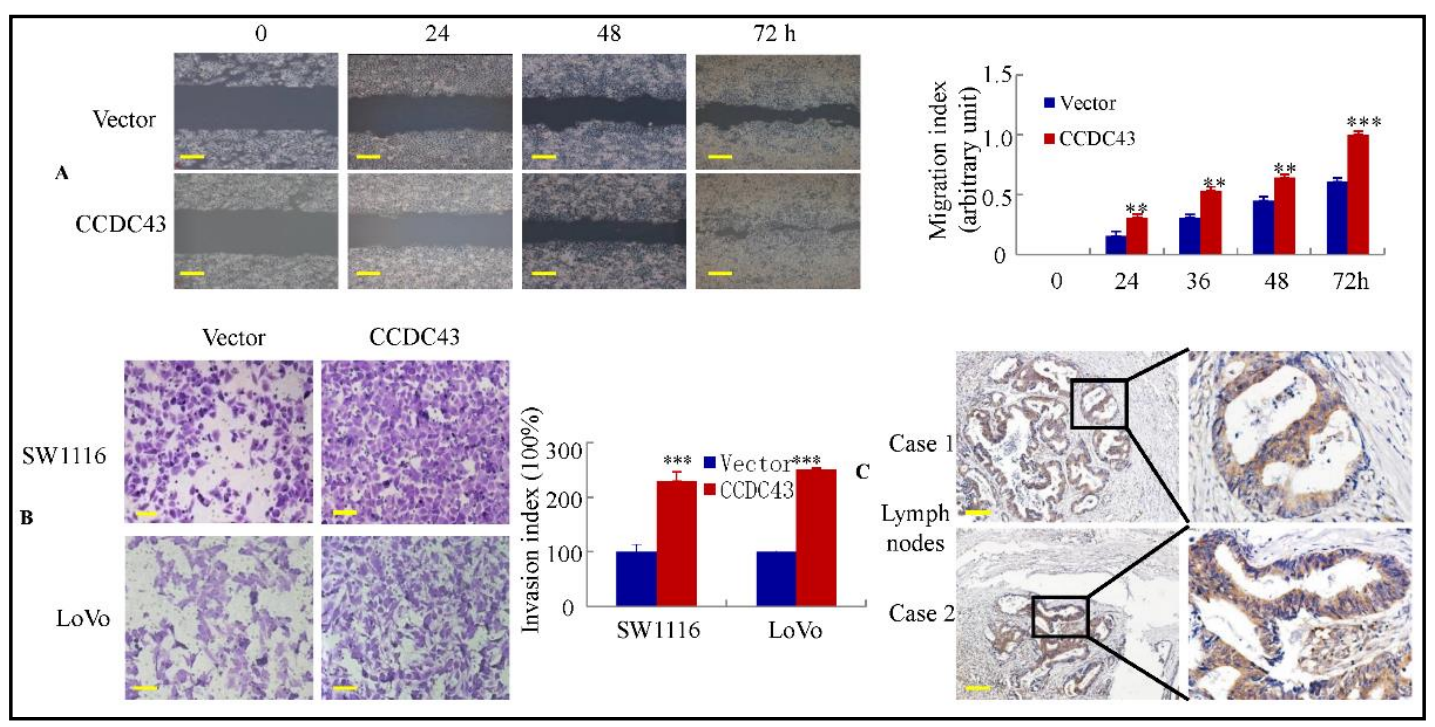

Fig. 3. High expression of protein CCDC43 is associated with the aggressive malignant behavior of CRC. (A) \& (B) CCDC43 overexpression significantly promoted migration and invasive ability compared with the vector. ${ }^{* *}, \mathrm{p}<0.05$ and ${ }^{* *}, \mathrm{p}<0.01$, vector vs. CCDC43. All experiments were repeated 3 times with identical findings. (C) Representative IHC images are shown for CCDC43 expression in lymph node metastatic cancer tissues. Scale bars, $100 \mu \mathrm{m}$ in A, $200 \mu \mathrm{m}$ in B and $100 \mu \mathrm{m}$ in C. 
a significantly increased percent closure at the $72 \mathrm{~h}$ endpoint (Fig. 3A). Similarly, the tumor invasion assay of CCDC43-overexpressing cells revealed an increase compared with the vector control cells (Fig. 3B).

Moreover, we detected the expression of CCDC43 in regional lymph nodes related to metastasis. In total, $10 / 10$ of the metastatic tissues collected from lymph nodes exhibited high CCDC43 expression by IHC, as exemplified in two patients (Fig. 3C). There was a correlation between the expression of CCDC43 in the primary lesion and metastasis to a regional lymph node.

The observed upregulated expression of CCDC43 in CRC prompted usto further investigate the clinical relevance of CCDC43 in the progression of CRC. The potential correlations of CCDC43 expression to a few clinicopathological parameters (Table 2) were examined in the patients. CCDC43 overexpression was associated with differentiation $(\mathrm{P}=0.025)$, American Joint Committee on Cancer (AJCC) stage I/ II vs. AJCC Stage III/IV (P $=0.039$ ), and lymph node metastasis $(\mathrm{P}=0.041)$. However, no associations were found between CCDC43 expression and other clinical features, including age, gender, tumor site and tumor location.

Table 2. Correlation between CCDC43 protein expression and the clinicopathological parameters of colorectal carcinoma

\begin{tabular}{|c|c|c|c|c|}
\hline \multirow{2}{*}{ Features } & \multirow{2}{*}{ Total number $(\mathrm{n}=82)$} & \multicolumn{3}{|c|}{$\begin{array}{c}\text { Expression of CCDC43 } \\
\end{array}$} \\
\hline & & Low expression(\%) & High expression (\%) & $P$ values \\
\hline \multicolumn{5}{|c|}{ Age(years) } \\
\hline$<60$ & 20 & $6(30 \%)$ & $14(70 \%)$ & \multirow{3}{*}{$\mathrm{P}=0.605$} \\
\hline$\geq 60$ & 62 & $15(24.2 \%)$ & $47(75.8 \%)$ & \\
\hline \multirow{2}{*}{\multicolumn{5}{|c|}{ Gender }} \\
\hline Male & 47 & $12(25.5 \%)$ & & \\
\hline Female & 35 & $9(25.7 \%)$ & $26(74.3 \%)$ & \multirow{2}{*}{$\mathrm{P}=0.985$} \\
\hline \multicolumn{4}{|c|}{ Differentiation } & \\
\hline Well & 12 & $7(58.3 \%)$ & $5(41.7 \%)$ & \multirow{3}{*}{$\mathrm{P}=0.025$} \\
\hline Moderate & 65 & $13(20 \%)$ & $52(80 \%)$ & \\
\hline Poor & 5 & $1(20 \%)$ & $4(80 \%)$ & \\
\hline \multicolumn{5}{|c|}{ AJCC stage } \\
\hline I, II & 39 & $14(35.9 \%)$ & $25(64.1 \%)$ & \multirow{2}{*}{$\mathrm{P}=0.039$} \\
\hline IIIIV & 43 & $7(16.2 \%)$ & $36(83.7 \%)$ & \\
\hline \multicolumn{5}{|l|}{ Location } \\
\hline $\mathrm{C}, \mathrm{A}, \mathrm{T}$ & 29 & $11(58.3 \%)$ & $18(62.1 \%)$ & \multirow{2}{*}{$\mathrm{P}=0.059$} \\
\hline $\mathrm{D}, \mathrm{S}, \mathrm{R}$ & 53 & $10(58.3 \%)$ & $43(81.1 \%)$ & \\
\hline \multicolumn{5}{|c|}{ Lymph node metastasis } \\
\hline no & 41 & $15(36.6 \%)$ & $26(63.4 \%)$ & \multirow{2}{*}{$\mathrm{P}=0.041$} \\
\hline yes & 41 & $6(14.6 \%)$ & $35(85.4 \%)$ & \\
\hline \multicolumn{5}{|c|}{ Tumor size } \\
\hline$<3$ & 5 & $1(20 \%)$ & $4(80 \%)$ & \multirow{2}{*}{$P=1.000$} \\
\hline$\geq 3$ & 77 & $20(26 \%)$ & $57(74 \%)$ & \\
\hline
\end{tabular}

Fig. 4. CCDC43 expression in CRC was associated with a poor prognosis. (A) Expression analysis of CCDC43 protein in CRC by IHC. (B) Kaplan - Meier survival analysis of overall survival in all patients, (C) patients at the early stage of CRC and (D) patients at the late stage of CRC according to CCDC43 expression. The logrank test was used to calculate P-values. Scale bars, $400 \mu \mathrm{m}$ in A.

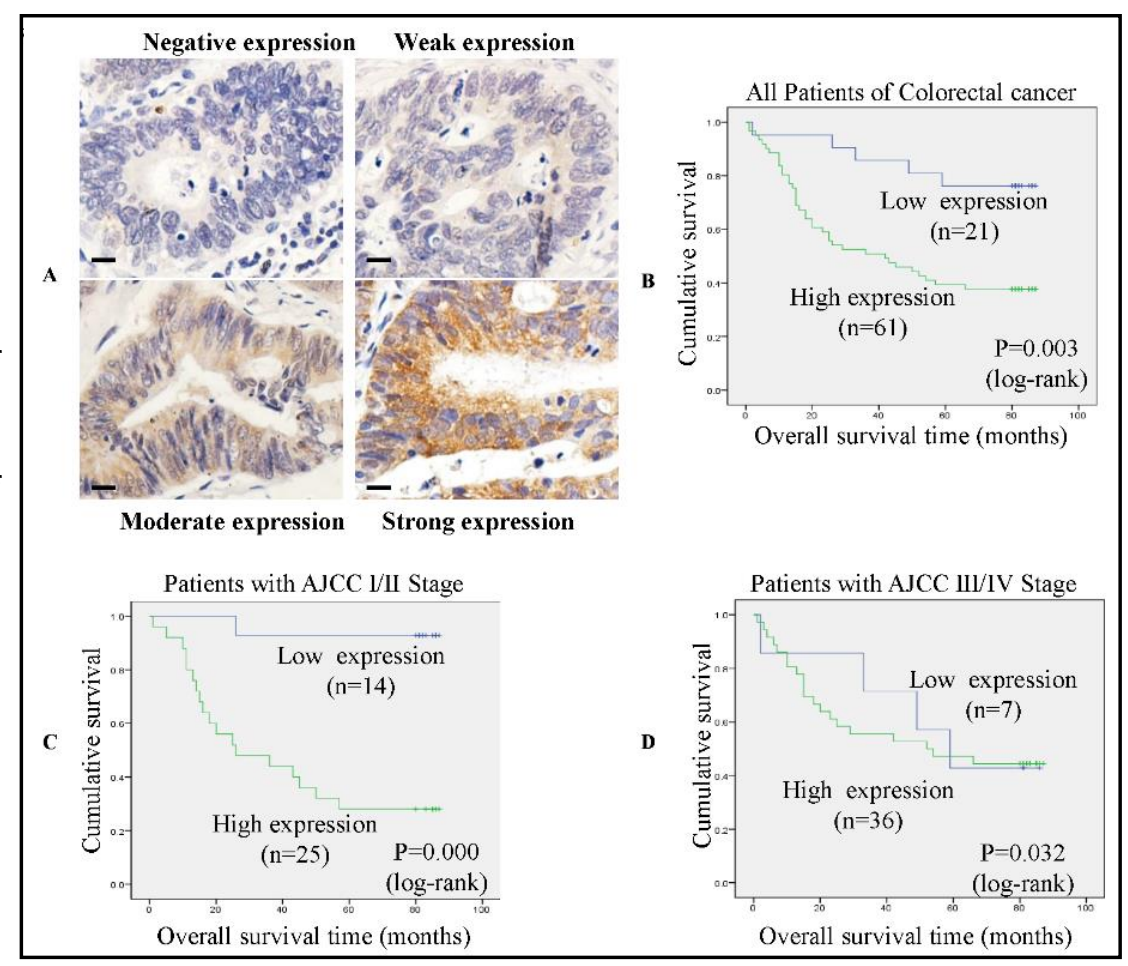


Furthermore, we evaluated the prognostic effect of CCDC43 on overall survival by comparing the overall survival of CRC patients with high (moderate and strong expression) or low (negative and weak expression) CCDC43 protein levels (Fig. 4A). Kaplan-Meier analysis indicated that patients with high CCDC43 expression had a shorter median survival time of 42 months. Patients with low CCDC43 expression had a median survival time of 81 months (Fig. 4B, $\mathrm{P}=0.003$ ). This relationship was more apparent in patients with early-stage CRC (AJCC stage I and II) (Fig. 4C, P = 0.000) than in those with late-stage CRC (AJCC stage III and IV) (Fig. 4D, $\mathrm{P}=0.032$ ). The prognostic value of CCDC43 expression in selective patient subgroups stratified according to AJCC stage was statistically significant.

The above findings suggested that increased CCDC43 expression significantly correlated with progression, metastasis, and poor outcome in CRC patients.

\section{TGF- $\beta$-induced upregulation of CCDC43 promoted metastasis through EMT in CRC}

EMT, one of the central mechanisms that induce the invasion and metastasis of tumors, is a process by which epithelial cells lose their polarity and are converted to a mesenchymal phenotype $[17,19,22]$. To investigate the effect of CCDC43 in EMT, we next examined the morphologic features of CRC cells. The stable vector-transfected cells displayed a polygonal cobblestone-like typical epithelial morphology in a confluent monolayer or islands of grouped cells with tight cell-cell contacts. However, CCDC43 transfectants had undergone a striking morphological change characterized by the loss of cell-cell contact and elongation, spindleshaped and scattered cells (Fig. 5A). These phenotypes are typical of the fibroblastoid cells formed during the EMT process.

Moreover, CCDC43-overexpresing cells were confirmed by Western blot analysis. EMT induction was demonstrated by a shift from the expression of epithelial markers (E-cadherin) to mesenchymal markers (vimentin; Fig. 5B).

Others have revealed an upregulation of transforming growth factor- $\beta$ (TGF- $\beta$ ) with human CCDC43 in normal ovarian fibroblast NOF151 cells using the Gene Expression Omnibus (GEO) database (https://www.ncbi.nlm.nih.gov/geoprofiles/123013665). Many studies have shown that TGF- $\beta$ can induce EMT $[23,24]$. Here, we aimed to explore whether CCDC43 was involved in TGF- $\beta 1$-induced EMT in CRC cells.

We showed that TGF- $\beta 1$ markedly induced the expression of the EMT marker vimentin and that CCDC43 was involved in TGF- $\beta 1$-induced EMT in CRC cells (Fig. 6A). Blocking TGF$\beta 1$ with its neutralizing antibody significantly suppressed endogenous CCDC43 expression and TGF- $\beta 1$-induced CCDC43 expression in LoVo cells (Fig. 6B). However, pre-transfection of the cells with CCDC43 siRNA not only inhibited CCDC43 expression but also suppressed TGF- $\beta 1$-induced vimentin expression (Fig. 6C).

Furthermore, CCDC43 siRNA neutralized the influence of TGF- $\beta 1$ on cell phenotypes (Fig. 6D). Coupled with the morphologic changes in EMT, CCDC43 knockdown decreased the invasive ability of tumor cells (Fig. 6E).

Fig. 5. CCDC43 regulated epithelialto - m e s e n chy mal transition (EMT) in vitro. (A) Morphology of stable Vector and CCDC43 transfectants visualized using phasecontrast microscopy. (B) EMT biomarkers were detected using

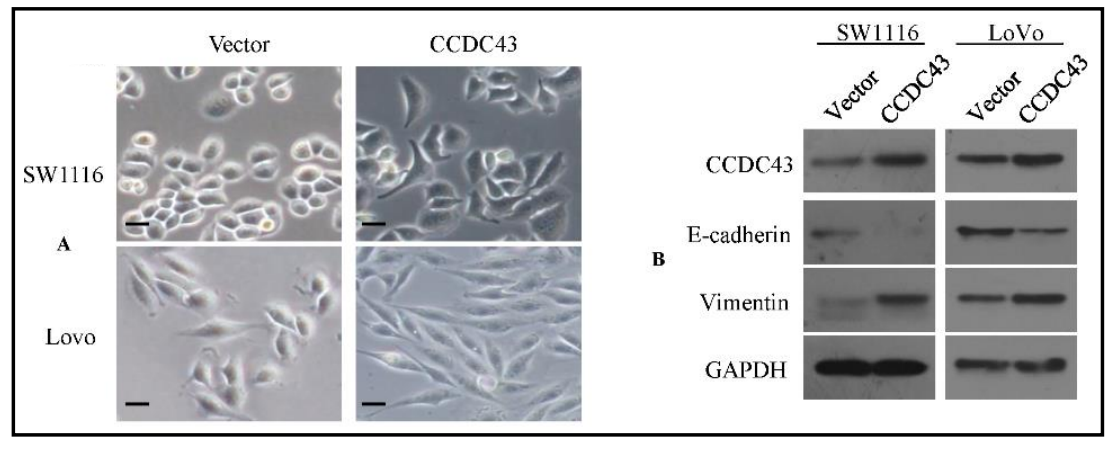
Western blot analysis $48 \mathrm{~h}$ after transfection. Scale bars, $100 \mu \mathrm{m}$ in A. 


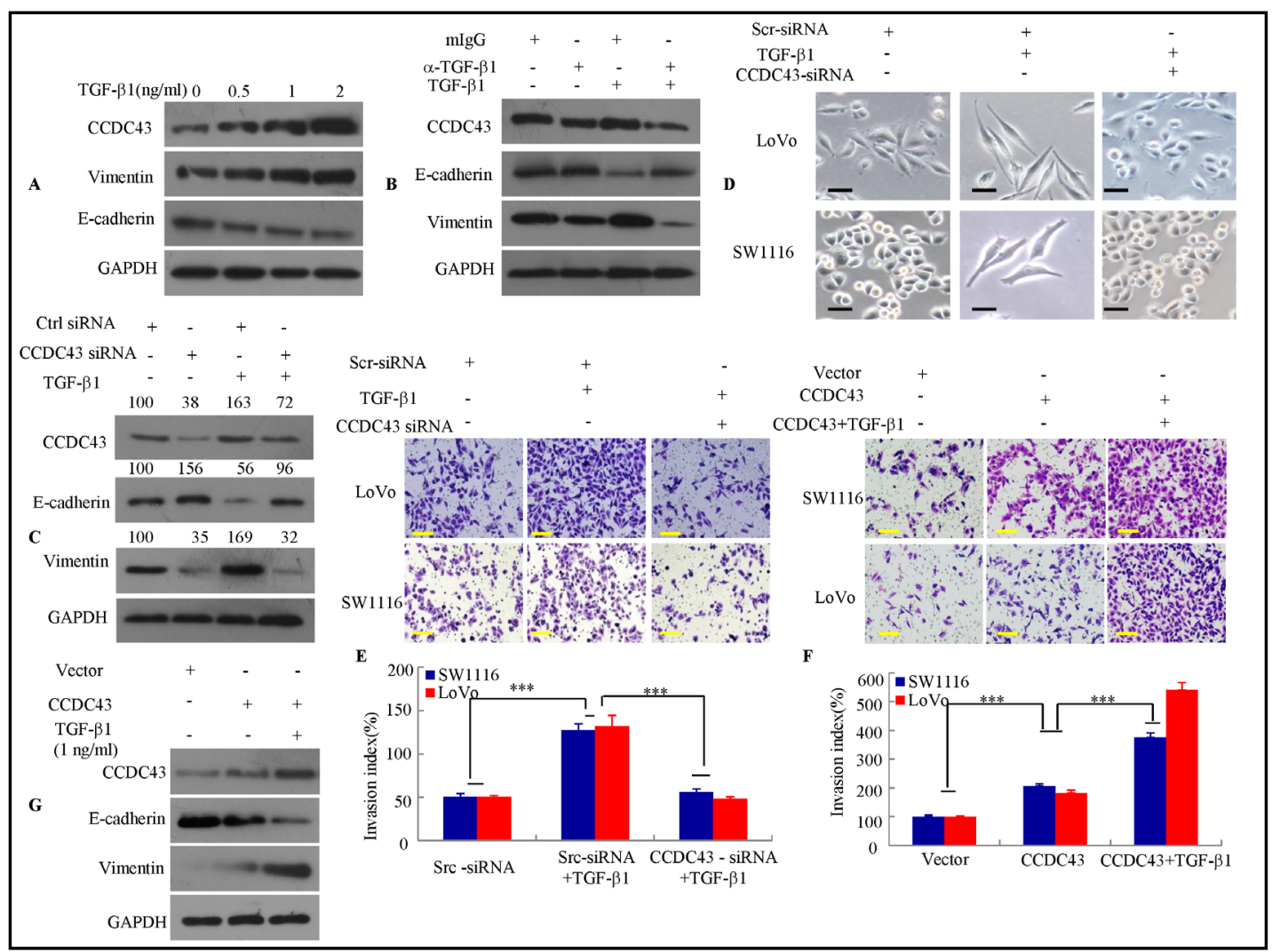

Fig. 6. CCDC43 is required for TGF- $\beta 1$-induced EMT and cell migration in CRC. (A) CCDC43 expression in LoVo cells treated with the indicated concentrations of recombinant TGF- $\beta$ for $48 \mathrm{~h}$ was detected by Western blot analysis with GAPDH as the internal control. (B) LoVo cells were treated with recombinant TGF- $\beta 1$ $(2 \mathrm{ng} / \mathrm{ml}$ ) in the presence of neutralizing anti-TGF- $\beta 1$ antibody ( $\alpha$-TGF- $\beta, 2 \mu \mathrm{g} / \mathrm{ml}$ ) or mouse IgG (mIgG) for $48 \mathrm{~h}$. (C) CCDC43 siRNA or Src siRNA were seeded in 6-well plates overnight and treated with TGF- $\beta 1$ ( $2 \mathrm{ng} / \mathrm{ml}$ ) for an additional $48 \mathrm{~h}$. The expression of CCDC43, E-cadherin and vimentin was detected by Western blotting. (D) CRC cell morphology was observed under an inverted microscope. (E) Representative images and transwell assay data for LoVo and SW1116 cells. Each bar represents the mean \pm SD. ***, P < 0.01 compared with findings in the absence of TGF- $\beta 1$. ***, P $<0.01$ in LoVo and SW1116 compared with cells transfected with CCDC43-siRNA and treated with TGF- $\beta 1$. (F) Vector and CCDC43-overexpressing stable transfectants by TGF- $\beta$ treatment were evaluated by transwell chamber assay. ${ }^{* * *} \mathrm{P}<0.01$, vector vs CCDC43, ${ }^{* * *} \mathrm{P}<0.01$, CCDC43 vs CCDC43 + TGF- $\beta 1$. The experiments were repeated at least three times. (G) The expression of E-cadherin and vimentin was detected by Western blotting using GAPDH as the internal control. Scale bars, $100 \mu \mathrm{m}$ in D; $200 \mu \mathrm{m}$ in E and F.

Besides, we examined that the invasion of CCDC43-overexpression cells in response to TGF- $\beta 1$. The results revealed that the over- expression of CCDC43 by the co-treatment of TGF- $\beta 1$ significantly augmented the invasive ability of CRC cells (Fig. 6F). In addition, CCDC43overexpressing cells in the presence of TGF- $\beta 1$ treatment were upregulated expression of vimentin, whereas were obviously downregulated expression of E-cadherin in CRC cells using western blot (Fig. 6G).

Together, these findings suggested that the induction of EMT by CCDC43 occured through TGF- $\beta 1$ signaling pathway.

\section{CCDC43 is direct transcriptional activation target of FOXK1}

We recently found that FOXK1 promoted EMT, invasion and metastasis in CRC cancer cell $[17,20]$. We assessed the correlation between CCDC43 and FOXK1 in CRC cancer cells. First, we evaluated the possible association between CCDC43 and FOXK1 expression in human 
CRC tissues. We analyzed the expression of CCDC43 and FOXK1 in ten freshly collected CRC biopsies. Western blot analyses indicated that both CCDC43 and FOXK1 were significantly upregulated throughout the examined tumor samples compared with the paired adjacent noncancerous tissues from the same patients (Fig. 7A). Spearman correlation analysis showed a positive correlation between CCDC43 and FOXK1 expression (correlation coefficient $r=$ $0.860, \mathrm{P}<0.000$, Fig. 7B).

Previous reports have suggested that the transcription factor FOXK1, which mediates its DNA binding, recognizes the DNA sequence motif, WRTAAAAYA $[15,18]$. We evaluated whether FOXK1 regulated CCDC43 transcription by surveying CCDC43 in the proximal promoter $(<800 \mathrm{bp})$ regions and identified the following two potential FOXK1 binding sites: site $1,-498$ to -490 and site $2,-677$ to -669 (numbering is from the transcription start site; Fig. 7C).

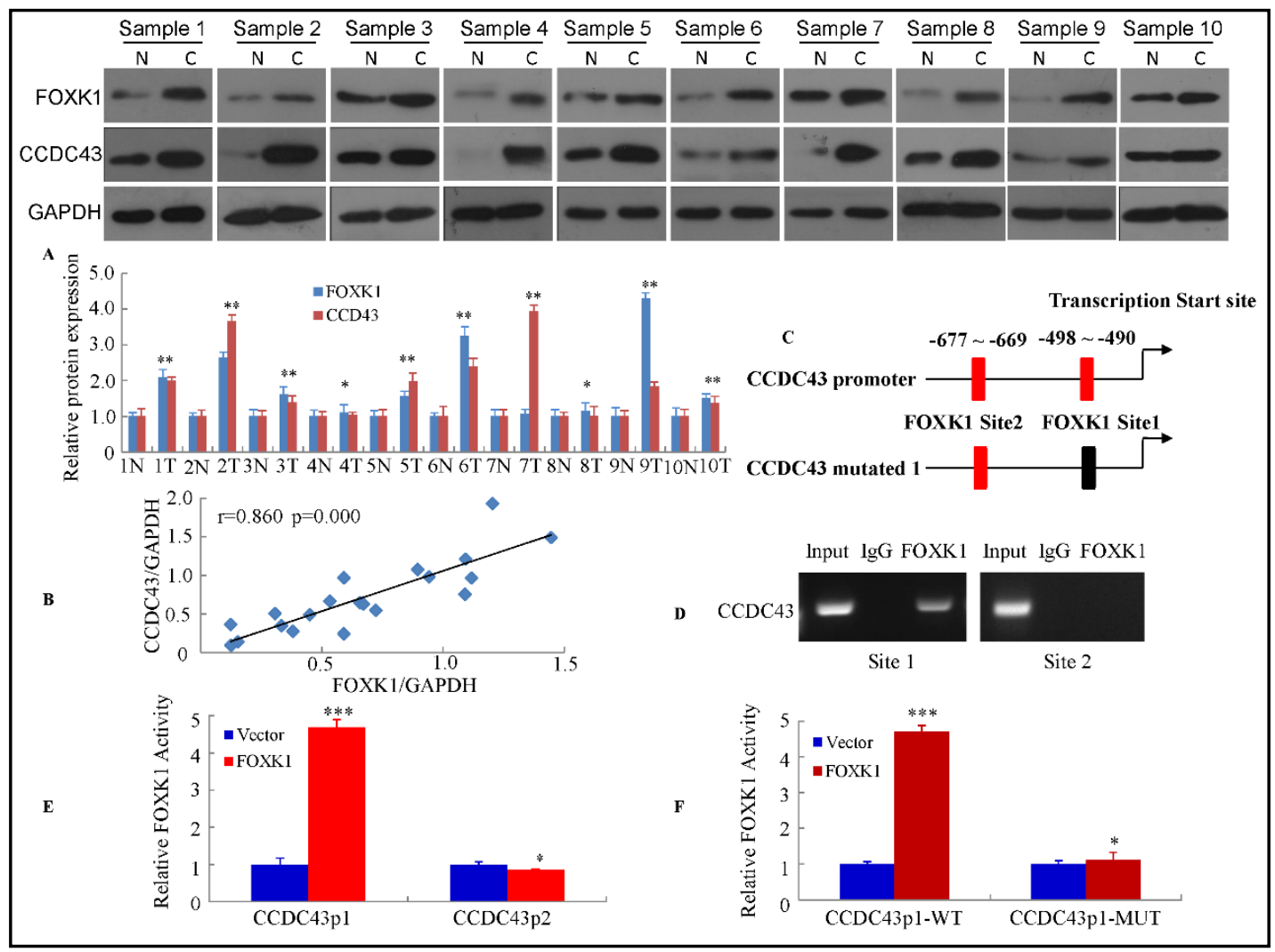

Fig. 7. FOXK1 binds directly to the promoter of CCDC43. (A) Western blot examination of CCDC43 and FOXK1 protein expression in ten freshly collected CRC biopsies. The relative protein expression levels were quantified by comparing the gray level of each band using Quantity One Software (Life Science Research, Hercules, CA, USA). (B) Spearman's correlation analysis was used to determine the relationship between CCDC43 and FOXK1 protein expression. (C) The luciferase (Luc) reporter constructs contained the CCDC43 promoter (CCDC43p) with a potential FOXK1 binding site upstream of the luciferase gene. wt, wild-type; mut, mutated. (D) Chromatin immunoprecipitation (ChIP) was performed using an anti-FOXK1 antibody or control IgG in LoVo cells. The CCDC43 promoter region to which FOXK1 binds was significantly enriched after immunoprecipitation with an anti-FOXK1 antibody. PCR products following ChIP were run on an ethidium-stained gel. (E) The reporter constructs used in the FOXK1 transfection experiments. FOXK1 activates CCDC43p1 and CCDC43p2 after co-transfection in LoVo cells. Luciferase activity was measured $48 \mathrm{~h}$ after transfection. Luciferase activity is expressed as the ratio of the promoter reporter activity to the control vector luciferase activity. ${ }^{* * *} \mathrm{P}<0.01$ and ${ }^{*} \mathrm{P}>0.05$. (F) Site-directed mutagenesis of CCDC43p1 was performed to generate CCDC43p-MUT. ${ }^{* * *} \mathrm{P}<0.01$ and ${ }^{*} \mathrm{P}>0.05$. 
To determine whether FOXK1 binds to the CCDC43 promoter region in vivo, we performed ChIP analysis using an anti-FOXK1 antibody in LoVo cells. PCR amplification revealed the immunoprecipitation of a 210-bp band containing the first possible binding site 1. Normal rabbit IgG was used as the negative control. No bands were evident in the immunoprecipitates for the other possible binding site 2 or for the control IgG (Fig. 7D).

Next, we cloned the promoter regions FOXK1-p498 (CCDC43p1) and FOXK1-677 (CCDC43p2) of human FOXK1 upstream of a luciferase gene in a reporter plasmid. The dual-luciferase assay showed that the activity of CCDC43p1 in FOXK1 cells increased 4.69fold compared with the vector cells, whereas the magnification exhibited a slight increase following CCDC43p2 transfection (Fig. 7E). Site-directed mutagenesis showed that the CCDC43p1 binding sites were critical for FOXK1-induced CCDC43 transactivation (Fig. 7F). These studies suggested that FOXK1 transactivated CCDC43 expression.

\section{CCDC43 is necessary for FOXK1-mediated EMT and metastasis in vitro}

To investigate whether the mechanism by which FOXK1 regulated CCDC43 plays a role in cell invasion and metastasis, we downregulated CCDC43 in FOXK1- overexpressing cells using siRNA and confirmed this effect by Western blot analysis (Fig. 8A). Next, we performed a wound-healing and transwell wassay. Knockdown of CCDC43 in FOXK1-overexpressing cells led to reduced migratory and invasive potential of FOXK1-overexpressing cells in vitro (Fig. 8 B \& C). Next, the expression of a typical EMT epithelial marker, E-cadherin, was upregulated after knockdown of CCDC43 in FOXK1-overexpressing cells. In contrast, the mesenchymal markers MMP2 and Vimentin were all downregulated (Fig. 9A). Immunofluorescence

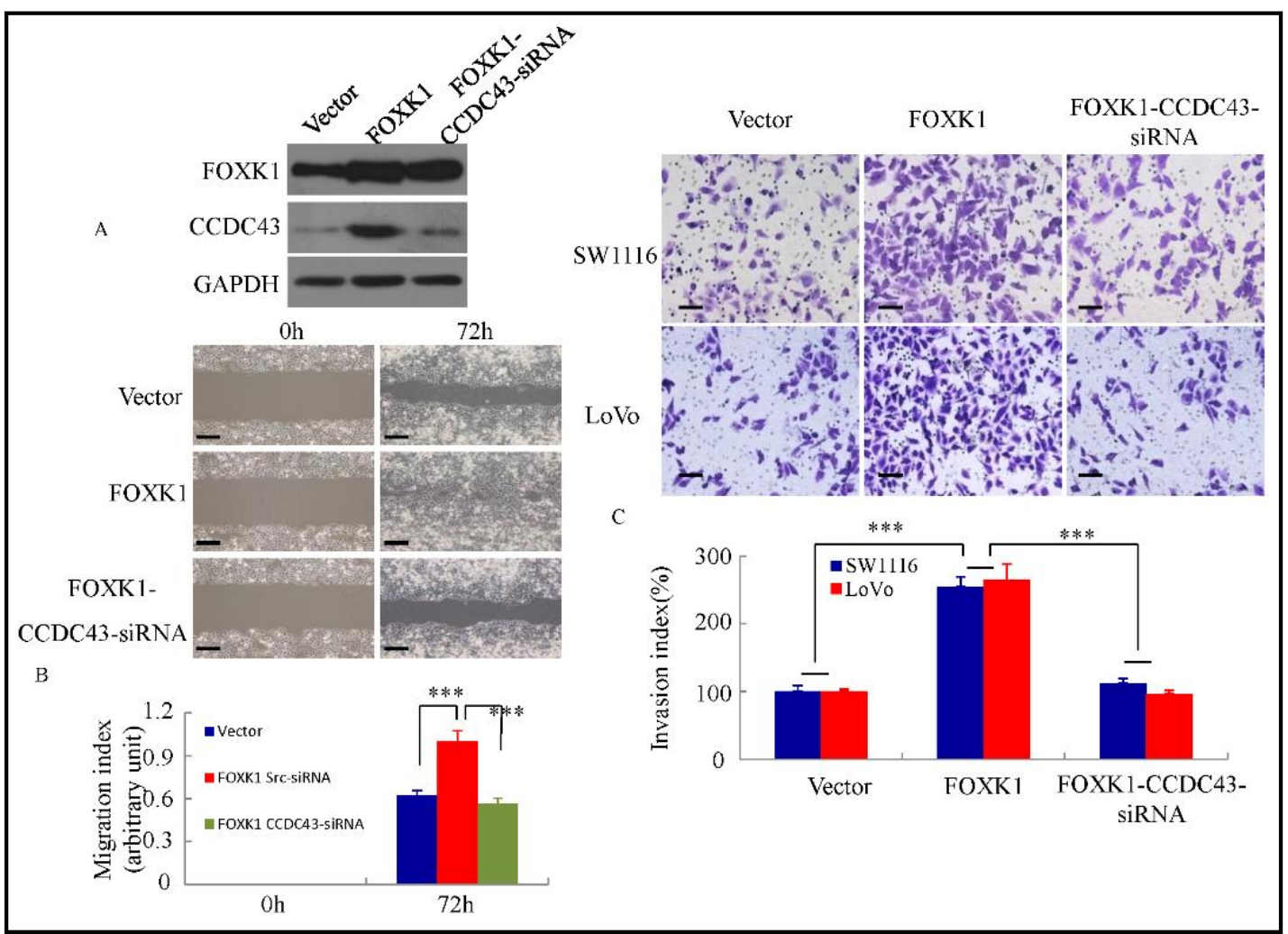

Fig. 8. CCDC43 is critical for FOXK1-mediated metastasis. (A) Expression levels of CCDC43 were detected by Western blot analysis in LoVo cells transfected with FOXK1-overexpressing plasmids followed by CCDC43 siRNA or Scr siRNA as a negative control. (B) For the wound-healing experiments, the cells were analyzed by live-cell microscopy. ${ }^{* * *} \mathrm{P}<0.01$. (C) Vector, stable FOXK1 transfectants were transfected with CCDC43 siRNA $48 \mathrm{~h}$ later, and the invasive ability of the cells decreased. ${ }^{* * *} \mathrm{P}<0.01$. The experiments were repeated at least three times. Scale bars, $100 \mu \mathrm{m}$ in B, $200 \mu \mathrm{m}$ in C. 
staining of E-cadherin and vimentin confirmed the EMT-associated shift in marker expression (Fig. 9B). Moreover, bioinformatics approaches for the analysis of the PhosphoSite database (http://www.phosphosite.org/ homeAction.action) revealed that FOXK1 had several potential ERK phosphorylation site, and the ERK1/2 pathway has been reported to be a key regulator of EMT $[25,26]$. Therefore, we performed a Western blot analysis to elucidate the phosphorylation status of proteins. FOXK1-overexpressing cells showed upregulated phosphorylation levels of ERK1/2 and AKT in comparison to the vector cells, whereas CCDC43 knockdown in FOXK1-overexpressing cells resulted in downregulation compared with FOXK1-overexpressing cells with intact CCDC43. Furthermore, the total amount of ERK1/2 and AKT protein was unaltered (Fig. 9C). Thus, FOXK1 overexpression promoted EMT by regulating CCDC43 through activation of the ERK1/2 and AKT pathway.

In addition, we detected CCDC43 and FOXK1 expression in regional lymph node metastatic CRC tissue and found that CCDC43 and FOXK1 were expressed at high levels in cancer cells (Fig. 9D). This result confirmed the positive correlation between CCDC43 and FOXK1 that was observed by IHC.

Taken together, these data suggest a critical role for CCDC43 in the induction of EMT and in the migration and invasion phenotypes caused by abnormal FOXK1 expression.

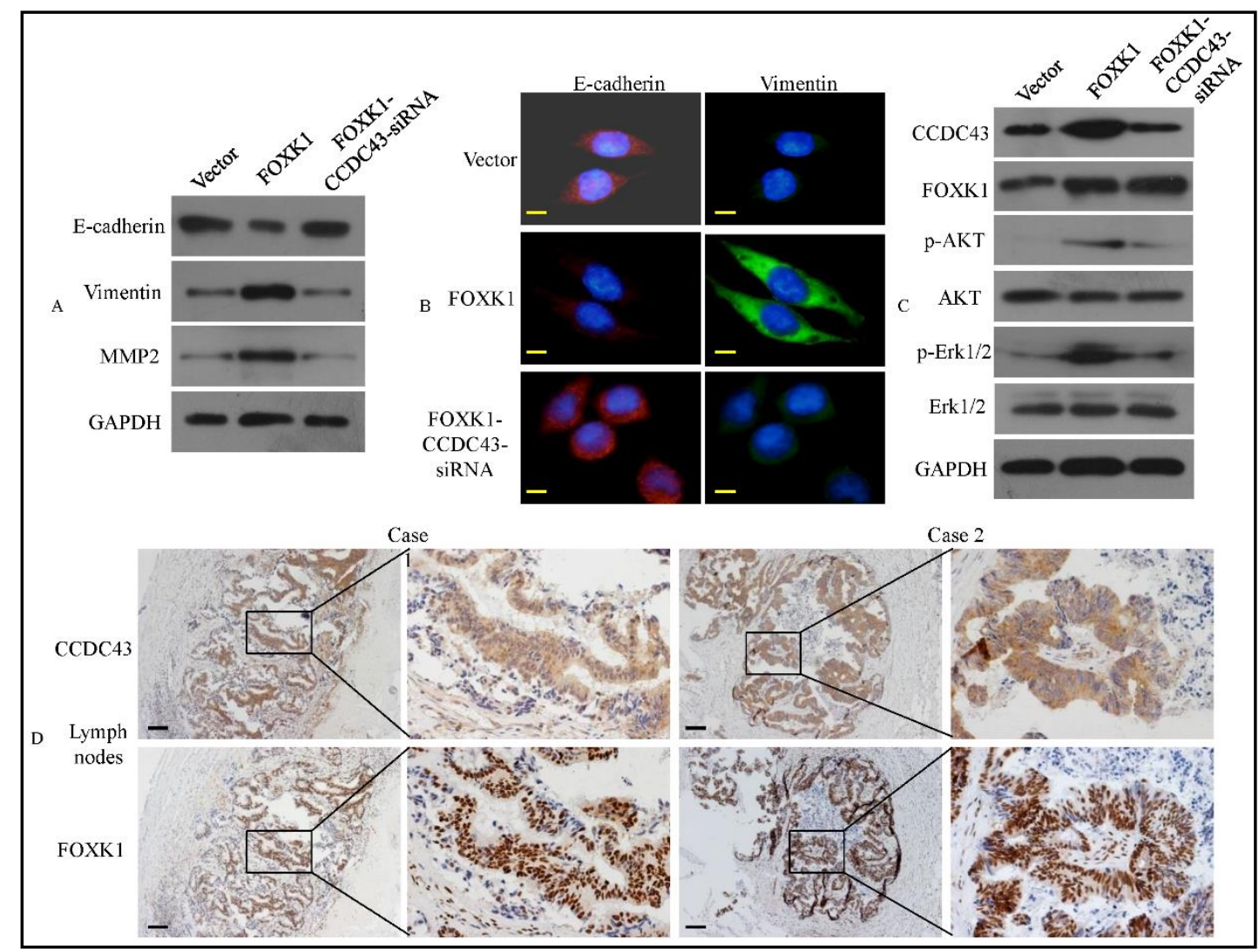

Fig. 9. CCDC43 is critical for FOXK1-mediated EMT and metastasis in vitro. (A) EMT biomarkers, including E-cadherin, vimentin and MMP2, were detected by Western blot analysis $48 \mathrm{~h}$ after transfection. (B) Immunofluorescence and microscopic visualization of E-cadherin (red) and vimentin (green). (C) CCDC43 repression inhibited the AKT/ERK/EMT signaling pathway, as detected by Western blot analysis. The images are representatives of three independent experiments with identical results. (D) Representative IHC images are shown for CCDC43 and FOXK1 expression in lymph node metastatic cancer tissues. Scale bars, $400 \mu \mathrm{m}$ in $\mathrm{B}$ and $100 \mu \mathrm{m}$ in $\mathrm{D}$. 


\section{Cellular Physiology Cell Physiol Biochem 2018;51:2547-2563 and Biochemistry \begin{tabular}{l|l} 
DOI: 10.1159/000495924 & (c) 2018 The Author(s). Published by S. Karger AG, Basel \\
Publishw.karger.com/cpb
\end{tabular}

\section{CCDC43 is necessary for FOXK1-induced CRC metastasis in vivo}

To further study the function of CCDC43 and FOXK1 in the metastasis of CRC in vivo, a liver metastasis model was generated by injecting LoVo/EGFP-N1, LoVo/EGFP-FOXK1-scr shRNA and LoVo/EGFP-FOXK1-CCDC43 shRNA cells expressing the green fluorescent protein (GFP) into mouse spleens. Thirty days after injection, the mice with FOXK1-overexpressing LoVo cells, but not vector LoVo cells, formed a variety of large metastatic hepatic tumor nodules (Fig. 10A), whereas the mice with CCDC43 knockdown in FOXK1-overexpressing cells showed significantly inhibited formation of hepatic tumor nodules compared with those with scr shRNA in FOXK1-overexpressing cells. The presence of liver metastases from CRC was confirmed by histological analysis (Fig. 10B).

Therefore, we applied immunohistochemistry on mouse tumor tissue samples to test the expression of the mesenchymal marker Vimentin. Overexpression of FOXK1 resulted in significant upregulation of vimentin in the IHC assay (Fig. 10C), whereas the loss of CCDC43 in FOXK1-overexpressing cells caused a decrease in vimentin expression, as determined by qRT-PCR (Fig. 10D). Cumulatively, our results suggested that CCDC43 was necessary for FOXK1-mediated EMT and metastasis in vivo.

\section{Discussion}

In the present study, we demonstrated that CCDC43 was involved in the tumorigenesis and metastasis of CRC. CCDC43 was overexpressed in cancer tissues and furthermore, for the first time, the role of CCDC43 in promoting EMT was identified. Moreover, our results showed that the functional region of the CCDC43 promoter was bound by FOXK1 to regulate the gene expression of CCDC43. Therefore, these data indicate that the cooperative relationship between CCDC43 and FOXK1 plays a pivotal role in the development or tumor progression.

Previous studies have shown genetic or epigenetic alterations in several CCDC genes in human cancers, including CCDC19 (NESG1) in nasopharyngeal carcinoma [7], CCDC62

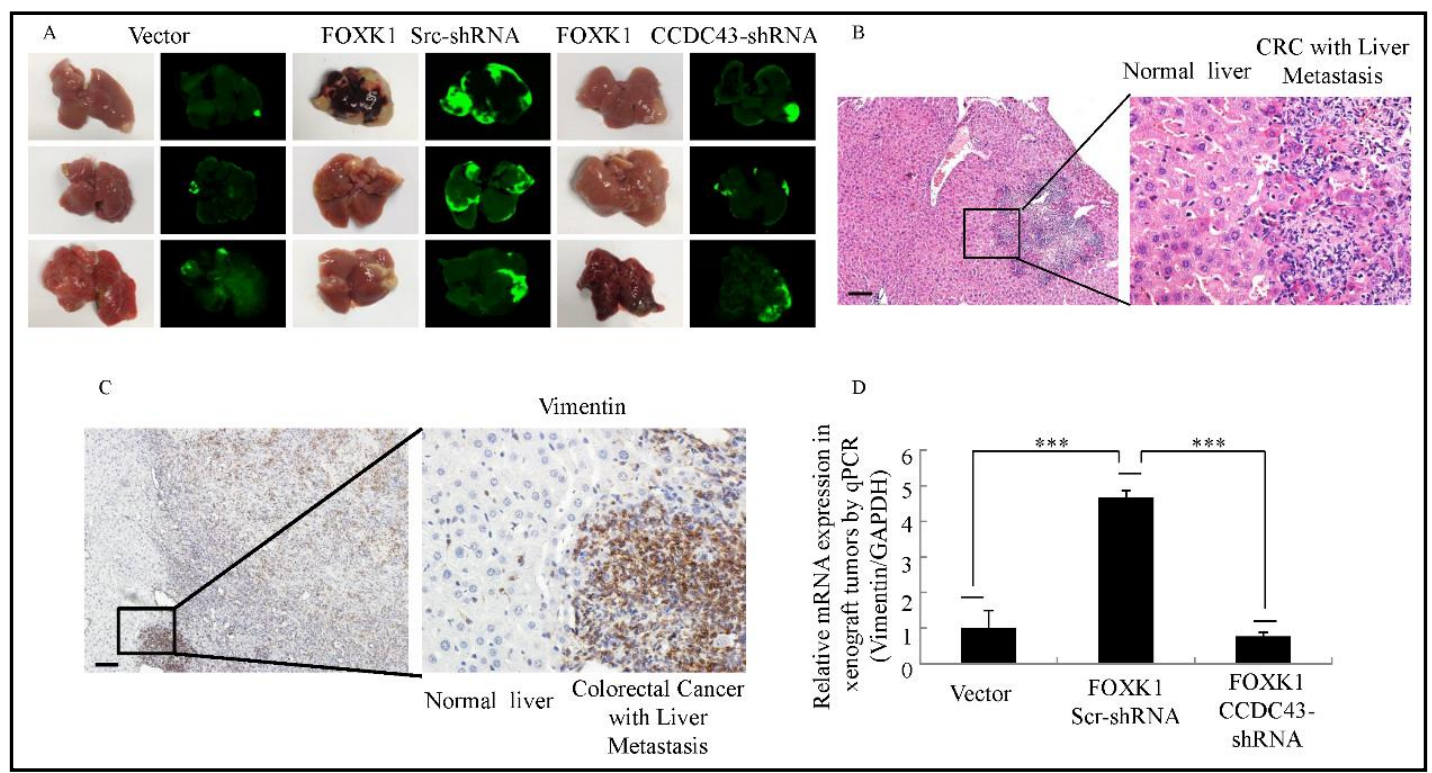

Fig. 10. CCDC43 synergizes with FOXK1 to promote tumor metastasis in vivo. (A) External whole-body fluorescence images of the liver by injection of LoVo/ pEGFP-N1(Vector), LoVo/pEGFP-FOXK1-scr-shRNA and LoVo/pEGFP-FOXK1- CCDC43-shRNA were obtained 30 days after spleen injection. The mice were sacrificed. (B) Metastatic cancer tissues were stained with H\&E. (C) Vimentin expression was detected in the CRC-derived liver metastases by IHC. (D) Vimentin expression in tumors derived from LoVo cells was measured by qRT-PCR; *** $\mathrm{P}<0.01$. Scale bars, $100 \mu \mathrm{m}$ in $\mathrm{B}$ and $\mathrm{C}$. 
in prostate cancer [5], CCDC116 in pancreatic cancer [6]. We reported the newly identified coiled-coil domain-containing CCDC43 gene in CRC cancer. The GENT database shows that CCDC43 mRNA is upregulated in CRC. We confirmed that CCDC43 is overexpressed in CRC cells and tissue. However, CCDC43 expression and its relationship with clinicopathological characteristics and the prognosis of patients with CRC remain largely unknown. In the present study, we showed a significant correlation between elevated CCDC43 expression and the TNM stage, AJCC stage, differentiation and lymph node metastasis. These strong correlations suggest that the high CCDC43 expression correlated to tumor invasion and lymph node metastasis in CRC. Kaplan-Meier analysis of the survival curves showed a significantly worse overall survival for patients whose tumors had high CCDC43 levels. Therefore, CCDC43 is a novel prognostic biomarker for patients with CRC.

Metastasis is a multistep process that involves the dissemination of cells from the primary tumor and colonization of distant secondary organs. Epithelial cells at the invasive front of a carcinoma acquire an enhanced migratory phenotype in a process called EMT. This cellular plasticity seems to drive the initiation of metastasis $[21,22]$. In our previous study, we found that overexpression of FHL2 in CRC cells was essential for maintaining their malignant phenotype and might be a mediator or a trigger of EMT in CRC cells [23]. Similarly, CCDC43 overexpression induced EMT, causing a loss of epithelial polarity. A stable transfectant of CCDC43 promoted migration, metastasis and dissemination, thereby facilitating tumor development and progression in CRC cells. These findings imply that aberrant CCDC43 upregulation might be an important mechanism underlying cancer metastasis.

TGF- $\beta 1$, an EMT stimulant, is a multifunctional cytokine that regulates a wide range of cellular functions $[17,19]$. TGF- $\beta 1$-stimulated cells become spindle-shaped and undergo morphological changes such as a decrease in cell-cell adhesion and loss of polarity. TGF- $\beta 1$ has also been shown to promote tumor progression and metastasis in established cancers, in part through the induction of EMT $[19,23]$. For example, Zhou Q et al. showed that the TGF- $\beta$ pathway regulates the EMT of gastric cancer cells by increasing the levels of miRNA$181 \mathrm{~b}$ to target Timp3 via the Smad2/3/4-dependent pathway [27]. While investigating the mechanistic interactions between TGF- $\beta 1$ and CCDC43 signaling during induction of EMT in CRC, we found that induction of EMT in CRC cells by TGF- $\beta 1$ was associated with a significant increase in CCDC43 expression. When CCDC43 expression was silenced, TGF- $\beta 1$ induction of the EMT phenotype was abrogated, and CCDC43 expression was also decreased. Our results suggest that CCDC43 might act as a potent inducer of EMT in CRC cells.

The FOXK1 proto-oncogene transcription factor is a member of the forkhead family. Wang and colleagues reported that FOXK1 protein levels elevated in human CRC [28]. Our previous studies have shown that induction of EMT in gastrointestinal cancers cells by TGF- $\beta 1$ was associated with a significant increase in FOXK1 expression. TGF- $\beta 1$ induced changes in morphology, significantly decreases E-cadherin expression and concomitantly increased vimentin expression. Our findings suggest FOXK1 acts as a co-stimulator in TGF- $\beta 1$-induced EMT in gastrointestinal cancers $[17,20]$. Here, CCDC43 served as a mediator of EMT inducer and the induction of EMT by CCDC43 was TGF- $\beta$ dependent. Thus, expressions of CCDC43 and FOXK1 are involved in TGF- $\beta 1$ signaling in CRC. Moreover, we analyzed potential correlations between CCDC43 and FOXK1 expression in human CRC tissues. Spearman correlation analysis revealed a positive correlation between CCDC43 and FOXK1 expression. Our novel finding that FOXK1 might upregulate the expression of CCDC43 facilitates an improved understanding of the mechanisms downstream of CCDC43 that regulate CRC cells. We then investigated whether CCDC43 was directly regulated by FOXK1. By chromatin immunoprecipitation and luciferase analysis, we showed evidence that FOXK1 bound to the CCDC43 promoter and directly induced CCDC43 expression, leading to invasion and EMT events. Therefore, we speculated that the FOXK1- CCDC43 axis promoted EMT, invasion and metastasis of CRC cells. 


\section{Cellular Physiology Cell Physiol Biochem 2018;51:2547-2563 and Biochemistry \begin{tabular}{l|l} 
DOI: 10.1159/000495924 & $\begin{array}{l}\text { @ } 2018 \text { The Author(s). Published by S. Karger AG, Basel } \\
\text { www.karger.com/cpb }\end{array}$
\end{tabular}}

Wang et al.: FOXK1 Promotes CCDC43-Mediated CRC Progression

\section{Conclusion}

In conclusion, our study shows that CCDC43 is upregulated in primary colon tumors and induces EMT and metastasis. FOXK1 transactivates CCDC43 expression by directly binding to its promoter. Thus, signaling pathway involving the FOXK1-CCDC43 axis may play crucial role in the progression of CRC and may lead to develop potential drugs for the treatment of the CRC.

\section{Acknowledgements}

This study was supported by grants from the National Natural Science Funds of China (grant numbers 81672875 \& 81772964), 'President Foundation of Nanfang Hospital, Southern Medical University' (grant numbers 2012B009, 2013Z007), and high-level topicmatching funds of Nanfang Hospital (grant numbers 201347 and G201227). Guangdong Provincial Key Laboratory of Gastroenterology, Department of Gastroenterology, Nanfang Hospital, Southern Medical University; Guangzhou Pilot Project of Clinical and Translational Research Center (early gastrointestinal cancer, grant number 7415696196402).

\section{Disclosure Statement}

The authors declare no conflict of interests.

\section{References}

1 Sohn RL, Vikstrom KL, Strauss M, Cohen C, Szent-Gyorgyi AG, Leinwand LA: A 29 residue region of the sarcomeric myosin rod is necessary for filament formation. J Mol Biol 1997;266:317-330.

-2 Burkhard P, Stetefeld J, Strelkov SV: Coiled coils: a highly versatile protein folding motif. Trends Cell Biol 2001;11:82-88.

-3 Kokona B, Rosenthal ZP, Fairman R: Role of the coiled-coil structural motif in polyglutamine aggregation Biochemistry-Us 2014;53:6738-6746.

4 Liu PY, Liu YW, Lin LJ, Chen JH, Liao JK: Evidence for statin pleiotropy in humans: differential effects of statins and ezetimibe on rho-associated coiled-coil containing protein kinase activity, endothelial function, and inflammation. Circulation. 2009;119:131-138.

5 Chen M, Ni J, Chang HC, Lin CY, Muyan M, Yeh S: CCDC62/ERAP75 functions as a coactivator to enhance estrogen receptor beta-mediated transactivation and target gene expression in prostate cancer cells. Carcinogenesis 2009;30:841-850.

6 Tsolakis AV, Grimelius L, Islam MS: Expression of the coiled coil domain containing protein 116 in the pancreatic islets and endocrine pancreatic tumors. Islets 2012;4:349-353.

7 Liu Z, Chen C, Yang H, Zhang Y, Long J, Long X, Fang W: Proteomic features of potential tumor suppressor NESG1 in nasopharyngeal carcinoma. Proteomics 2012;12:3416-3425.

-8 Modjtahedi N, Tokatlidis K, Dessen P, Kroemer G: Mitochondrial Proteins Containing Coiled-Coil-HelixCoiled-Coil-Helix (CHCH) Domains in Health and Disease. Trends Biochem Sci 2016;41:245-260.

-9 Park SJ, Jang HR, Kim M, Kim JH, Kwon OH, Park JL, Noh SM, Song KS, Kim SY, Kim YH, Kim YS: Epigenetic alteration of CCDC67 and its tumor suppressor function in gastric cancer. Carcinogenesis 2012;33:14941501.

10 Naito D, Ogata T, Hamaoka T, Nakanishi N, Miyagawa K, Maruyama N, Kasahara T, Taniguchi T, Nishi M, Matoba S, Ueyama T: The coiled-coil domain of MURC/cavin-4 is involved in membrane trafficking of caveolin-3 in cardiomyocytes. Am J Physiol Heart Circ Physiol 2015;309:H2127-2136.

11 Kim H, Huang J, Chen J: CCDC98 is a BRCA1-BRCT domain-binding protein involved in the DNA damage response. Nat Struct Mol Biol 2007;14:710-715.

$\checkmark 12$ Bourne HR: Colon cancer: Consider the coiled coil.... Nature 1991;351:188-190. 


\section{Cellular Physiology Cell Physiol Biochem 2018;51:2547-2563

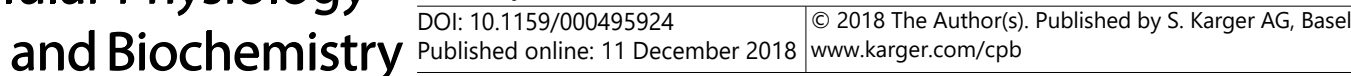 \\ Wang et al.: FOXK1 Promotes CCDC43-Mediated CRC Progression}

13 Garry DJ, Meeson A, Elterman J, Zhao Y, Yang P, Bassel-Duby R, Williams RS: Myogenic stem cell function is impaired in mice lacking the forkhead/winged helix protein MNF. Proc Natl Acad Sci U S A 2000; 97:5416-5421.

14 Yang Q Bassel-Duby R, Williams RS: Transient expression of a winged-helix protein, MNF-beta, during myogenesis. Mol Cell Biol. 1997;17:5236-5243.

15 Shi X, Seldin DC, Garry DJ: Foxk1 recruits the Sds3 complex and represses gene expression in myogenic progenitors. Biochem J 2012;446:349-357.

16 Yang Q Kong Y, Rothermel B, Garry DJ, Bassel-Duby R, Williams RS: The winged-helix/forkhead protein myocyte nuclear factor beta (MNF-beta) forms a co-repressor complex with mammalian sin3B. Biochem J 2000:335-343.

17 Wu Y, Peng Y, Wu M, Zhang W, Zhang M, Xie R, Zhang P, Bai Y, Zhao J, Li A, Nan Q Chen Y, Ren Y, Liu S, Wang J: Oncogene FOXK1 enhances invasion of colorectal carcinoma by inducing epithelial-mesenchymal transition. Oncotarget 2016;7:51150-51162.

18 Hawke TJ, Jiang N, Garry DJ: Absence of p21CIP rescues myogenic progenitor cell proliferative and regenerative capacity in Foxk1 null mice. J Biol Chem 2003;278:4015-4020.

19 Xie R, Wang J, Tang W, Li Y, Peng Y, Zhang H, Liu G, Huang X, Zhao J, Li A, Gong W, Chen Y, Ren Y, Wang Y, Li G, Liu S, Wang J: Rufy3 promotes metastasis through epithelial-mesenchymal transition in colorectal cancer. Cancer Lett 2017;390: 30-38.

20 Xie R, Wang J, Liu X, Wu L, Zhang H, Tang W, Li Y, Xiang L, Peng Y, Huang X, Bai Y, Liu G, Li A, Wang Y, Chen Y, Ren Y, Li G, Gong W, Liu S, Wang J: RUFY3 interaction with FOXK1 promotes invasion and metastasis in colorectal cancer. Sci Rep 2017;7:3709.

-21 Koo TH, Lee JJ, Kim EM, Kim KW, Kim HD, Lee JH: Syntenin is overexpressed and promotes cell migration in metastatic human breast and gastric cancer cell lines. Oncogene 2002;21:4080-4088.

-22 Yang MH, Wu MZ, Chiou SH, Chen PM, Chang SY, Liu CJ, Teng SC, Wu KJ: Direct regulation of TWIST by HIF1alpha promotes metastasis. Nat Cell Biol 2008;10:295-305.

23 Zhang W, Jiang B, Guo Z, Sardet C, Zou B, Lam CS, Li J, He M, Lan HY, Pang R, Hung IF, Tan VP, Wang J, Wong BC: Four-and-a-half LIM protein 2 promotes invasive potential and epithelial-mesenchymal transition in colon cancer. Carcinogenesis 2010;31:1220-1229.

24 Fan Y, Shen B, Tan M, Mu X, Qin Y, Zhang F, Liu Y: TGF- $\beta$-induced upregulation of malat1 promotes bladder cancer metastasis by associating with suz12. Clin Cancer Res 2014;20:1531-1541.

25 Wang Y, Lin Z, Sun L, Fan S, Huang Z, Zhang D, Yang Z, Li J, Chen W: Akt/ Ezrin Tyr353/NF-кB pathway regulates EGF-induced EMT and metastasis in tongue squamous cell carcinoma. Br J Cancer 2014;110:695705.

26 Zhou X, Hu Y, Dai L, Wang Y, Zhou J, Wang W, Di W, Qiu L: MicroRNA-7 inhibits tumor metastasis and reverses epithelial-mesenchymal transition through AKT/ERK1/2 inactivation by targeting EGFR in epithelial ovarian cancer. PLoS One 2014;9:e96718.

27 Zhou Q Zheng X, Chen L, Xu B, Yang X, Jiang J, Wu C: Smad2/3/4 Pathway Contributes to TGF- $\beta$-Induced MiRNA-181b Expression to Promote Gastric Cancer Metastasis by Targeting Timp3 Cell Physiol Biochem 2016;39:453-466

-28 Wang W, Li X, Lee M, Jun S, Aziz KE, Feng L, Tran MK, Li N, McCrea PD, Park JI, Chen J: FOXKs promote Wnt/ $\beta$-catenin signaling by translocating DVL into the nucleus. Dev Cell 2015;32:707-718. 